\title{
Potentially Functional Polymorphisms in POU5F1 Gene Are Associated with the Risk of Lung Cancer in Han Chinese
}

\author{
Rui Niu, Yuzhuo Wang, Meng Zhu, Yifan Wen, Jie Sun, Wei Shen, Yang Cheng, \\ Jiahui Zhang, Guangfu Jin, Hongxia Ma, Zhibin Hu, Hongbing Shen, and Juncheng Dai
}

Department of Epidemiology and Biostatistics, Jiangsu Key Lab of Cancer Biomarkers, Prevention and Treatment, Collaborative Innovation Center for Cancer Personalized Medicine, School of Public Health, Nanjing Medical University, Nanjing 211166, China

Correspondence should be addressed to Juncheng Dai; djc@njmu.edu.cn

Received 10 September 2015; Revised 28 November 2015; Accepted 6 December 2015

Academic Editor: Robert A. Vierkant

Copyright (c) 2015 Rui Niu et al. This is an open access article distributed under the Creative Commons Attribution License, which permits unrestricted use, distribution, and reproduction in any medium, provided the original work is properly cited.

\begin{abstract}
POU5F1 is a key regulator of self-renewal and differentiation in embryonic stem cells and may be associated with initiation, promotion, and progression in cancer. We hypothesized that functional polymorphisms in POU5F1 may play an important role in modifying the lung cancer risk. To test this hypothesis, we conducted a case-control study to explore the association between 17 potentially functional SNPs in POU5F1 gene and the lung cancer risk in 1,341 incident lung cancer cases and 1,982 healthy controls in a Chinese population. We found that variant alleles of rs887468 and rs3130457 were significantly associated with increased risk of lung cancer after multiple comparison $\left(\mathrm{OR}=1.29,95 \% \mathrm{CI}: 1.11-1.51, P_{\mathrm{fdr}}=0.017\right.$ for rs887468; OR $=1.29,95 \%$ CI: $1.10-1.51$, $P_{\mathrm{fdr}}=0.034$ for rs3130457, resp.). In addition, we detected a significant interaction between rs887468 genotypes and smoking status on lung cancer risk $(P=0.017)$. Combined analysis of these 2 SNPs showed a significant allele-dosage association between the number of risk alleles and increased risk of lung cancer $\left(P_{\text {trend }}<0.001\right)$. These findings indicate that potentially functional polymorphisms in POU5F1 gene may contribute to lung cancer susceptibility in a Chinese population.
\end{abstract}

\section{Introduction}

Lung cancer is the leading cause of cancer related mortality worldwide. Over $80 \%$ of lung cancer can be attributed to cigarette smoking [1]. However, Only $10 \%$ to $15 \%$ of chronic smokers develop lung cancer, indicating that other factors (e.g., genetic factors) might also play a pivotal role in lung cancer risk [2]. Recently, genome-wide association studies have discovered dozens of loci that are related to lung cancer risk [3-11]. These loci only account for a small fraction of the risk of developing lung cancer due to the stringent screening criteria of GWAS [8]. Thus, an effort on candidate gene strategies might help to explain the missing heritability.

The Pit-Oct-Unc (POU) homeodomain transcription factor, POU5F1 (also known as OCT-3, OCT-4, and OCT $3 / 4$ ), is a key regulator of self-renewal and differentiation in embryonic stem cells [12-15]. POU5F1 gene expresses in adult human stem cells, immortalized nontumorigenic cells, and tumor cells and cell lines, and its level decreases with the onset of differentiation and loss of pluripotency in these cells [1618]. According to the cancer stem cell (CSC) dogma, the reactivation of early embryonic stem cell genes such as POU5F1 in somatic stem cells and/or differentiating progenitor cells may lead to transformation into CSCs, which may result in cancer initiation, promotion, and progression [19-21]. To date, high expression level of POU5F1 has been detected in various types of cancer cells [22, 23]. In particular, Karoubi et al. observed higher levels of expression of POU5F1 gene and atypical cytoplasmic distribution of POU5F1 in lung adenocarcinoma cell lines, indicating an oncogenic role in lung adenocarcinoma [24]. Polymorphisms in POU class 5 homeobox 1 pseudogene 1 gene (POU5F1P1), a highly homologous pseudogene of POU5F1, were identified to be associated with the risk of gastric cancer [25]. Therefore, we postulated that potentially functional genetic variation within POU5F1 might modify the susceptibility to lung cancer. To test this hypothesis, we 
TABLE 1: Distributions of select variables in lung cancer cases and cancer-free controls.

\begin{tabular}{|c|c|c|c|}
\hline Variables & Case $(N=1,341)$ & Control $(N=1,982)$ & $P$ \\
\hline Age (mean \pm sd.) & $61.06 \pm 10.15$ & $61.32 \pm 11.07$ & 0.473 \\
\hline$\leq 60$ & $596(44.44 \%)$ & $883(44.55 \%)$ & \multirow{2}{*}{0.980} \\
\hline$>60$ & $745(55.56 \%)$ & $1099(55.45 \%)$ & \\
\hline \multicolumn{4}{|l|}{ Gender } \\
\hline Male & $949(70.77 \%)$ & $1358(68.52 \%)$ & \multirow{2}{*}{0.179} \\
\hline Female & $392(29.23 \%)$ & $624(31.48 \%)$ & \\
\hline \multicolumn{4}{|l|}{ Smoking status } \\
\hline Current & $634(47.28 \%)$ & $876(44.20 \%)$ & \multirow{3}{*}{$<0.001$} \\
\hline Former & $185(13.80 \%)$ & $86(4.34 \%)$ & \\
\hline Never & $522(38.92 \%)$ & $1020(51.46 \%)$ & \\
\hline \multicolumn{4}{|l|}{ Pack-year (py.) } \\
\hline$\leq 25$ & $774(57.72 \%)$ & $1505(75.93 \%)$ & \multirow{2}{*}{$<0.001$} \\
\hline$>25$ & $567(42.28 \%)$ & $477(24.07 \%)$ & \\
\hline \multicolumn{4}{|l|}{ Histology type } \\
\hline Squamous cell carcinoma & $481(35.87 \%)$ & & \\
\hline Adenocarcinoma & $860(64.13 \%)$ & & \\
\hline
\end{tabular}

conducted a case-control study including 1,341 cases and 1,982 controls to investigate the association between functional polymorphisms in POU5F1 and lung cancer risk.

\section{Materials and Methods}

2.1. Study Subjects. This case-control study was approved by the institutional review board of Nanjing Medical University. Cases were recruited from the Cancer Hospital of Jiangsu Province and the First Affiliated Hospital of Nanjing Medical University since 2003. Patients with histopathologically confirmed incident lung cancer were included. Exclusion criteria include having a prior history of other cancers, having metastatic cancer from other sites, or having undergone chemotherapy of radiotherapy. Controls were randomly selected from individuals participating in a community based noninfectious disease screening program in Jiangsu Province during the same time period. The controls were cancerfree and were frequency matched to cases by age and sex. We enrolled 1,341 cases and 1,982 controls in the final set. After providing a written informed consent, participants donated $5 \mathrm{~mL}$ venous blood sample and underwent a faceto-face interview that solicited information on participants' demographics (e.g., age and sex) and health related behaviors (e.g., smoking).

Those who had smoked one cigarette or more per day for $>1$ year were considered as smokers; smokers who had quit smoking for $>1$ year were defined as former smokers; all others were classified as never smokers [6]. Smoking dosage were measured by pack-years of smoking [(cigarettes per day/20) $\times$ smoking years]. In addition, smokers were divided into light and heavy smokers according to the threshold of 25 pack-years.

2.2. SNP Selection. All SNPs in the POU5F1 gene region and $10 \mathrm{~kb}$ upstream were screened based on the Han Chinese population (CHB) of the HapMap Project [HapMap Data Rel. 27 Phase II + III]. Minor allele frequency (MAF) $\geq 0.05$ was used to filter low-frequency variants. The remaining variants were annotated by SNPinfo Web Server (http://snpinfo.niehs .nih.gov/); 27 SNPs were selected as potentially functional variants. We then performed linkage disequilibrium (LD) analysis with an $r^{2}$ threshold of 0.8 ; 19 SNPs were retained for genotyping. However, rs1265163 and rs3132517 were excluded because of probe design failure. Therefore, there were 17 SNPs included in the final set (Table 2).

2.3. Genotyping. Genomic DNA was extracted from a leukocyte pellet by proteinase $\mathrm{K}$ digestion, followed by phenolchloroform extraction and ethanol precipitation. Illumina Infinium BeadChip (Illumina Inc.) was used for genotyping and GenTrain version 1.0 clustering algorithm in GenomeStudio V2011.1 (Illumina Inc.) for genotype calling. Technicians performing the genotyping were blinded to the case or control status of participants.

2.4. Statistical Analysis. Deviation of genotype distribution for each SNP from the Hardy-Weinberg equilibrium was tested by a goodness-of-fit $\chi^{2}$. Student's $t$ test for continuous variables and $\chi^{2}$ test for categorical variables were applied for analyzing distribution differences of demographic characteristics and genotypes between cases and controls. The association between SNPs and lung cancer risk was examined under an additive model using logistic regression to estimate odds ratios (ORs) [26] and 95\% confidence intervals (CIs) with adjustment for age, sex, and pack-years of smoking when appropriate. We used $\chi^{2}$-based $Q$-test to test the heterogeneity from corresponding subgroups. Multiplicative interactions were tested using a general logistic regression model by applying the equation

$$
Y=\beta_{0}+\beta_{G} G+\beta_{E} S+\beta_{G S}(G S)+\sum \beta_{i} \operatorname{Covar}_{i},
$$


TABLE 2: Summary of associations between 17 SNPs in selected POU5F1 gene with lung cancer risk.

\begin{tabular}{lccccccccc}
\hline SNP & Allele $^{\mathrm{a}}$ & Case $^{\mathrm{b}}$ & Control $^{\mathrm{b}}$ & Call rate $(\%)$ & MAF (case/control) & HWE $^{\mathrm{d}}$ & ${\text { OR }(95 \% \text { CI })^{\mathrm{e}}}^{\mathrm{P}^{\mathrm{e}}}$ & $P^{\mathrm{f}}$ \\
\hline rs887468 & G/A & $\mathbf{9 9 7 / 3 2 2 / 2 2}$ & $\mathbf{1 5 8 8 / 3 6 8 / 2 6}$ & $\mathbf{1 0 0 . 0 0}$ & $\mathbf{0 . 1 4 / 0 . 1 1}$ & $\mathbf{0 . 3 5}$ & $\mathbf{1 . 2 9}(\mathbf{1 . 1 1}-\mathbf{1 . 5 1})$ & $\mathbf{0 . 0 0 1}$ & $\mathbf{0 . 0 1 7}$ \\
rs3130457 & A/G & $\mathbf{1 0 3 0 / 2 9 3 / 1 8}$ & $\mathbf{1 6 2 6 / 3 3 5 / 2 1}$ & $\mathbf{1 0 0 . 0 0}$ & $\mathbf{0 . 1 2 / 0 . 1 0}$ & $\mathbf{0 . 4 3}$ & $\mathbf{1 . 2 9}(\mathbf{1 . 1 0}-\mathbf{1 . 5 1})$ & $\mathbf{0 . 0 0 2}$ & $\mathbf{0 . 0 3 4}$ \\
rs12215963 & A/G & $1102 / 221 / 8$ & $1694 / 254 / 9$ & 98.95 & $0.09 / 0.07$ & 1.00 & $1.26(1.05,1.53)$ & 0.015 & 0.255 \\
rs885948 & A/G & $321 / 665 / 355$ & $530 / 1000 / 452$ & 100.00 & $0.51 / 0.48$ & 0.65 & $1.13(1.02,1.25)$ & 0.022 & 0.374 \\
rs4713438 & G/A & $978 / 335 / 28$ & $1391 / 552 / 39$ & 100.00 & $0.15 / 0.16$ & 0.07 & $0.90(0.78,1.04)$ & 0.169 & 1.000 \\
rs2269713 & A/G & $875 / 411 / 55$ & $1337 / 579 / 66$ & 100.00 & $0.19 / 0.18$ & 0.70 & $1.09(0.96,1.24)$ & 0.181 & 1.000 \\
rs1052989 & G/A & $556 / 611 / 174$ & $772 / 931 / 279$ & 100.00 & $0.36 / 0.38$ & 0.96 & $0.93(0.84,1.04)$ & 0.201 & 1.000 \\
rs885950 & A/C & $463 / 636 / 241$ & $722 / 930 / 322$ & 99.73 & $0.42 / 0.40$ & 0.45 & $1.07(0.96,1.18)$ & 0.208 & 1.000 \\
rs879882 & A/G & $342 / 676 / 323$ & $553 / 964 / 465$ & 100.00 & $0.49 / 0.48$ & 0.26 & $1.06(0.96,1.18)$ & 0.227 & 1.000 \\
rs2394882 & C/A & $617 / 577 / 147$ & $851 / 914 / 217$ & 100.00 & $0.32 / 0.34$ & 0.23 & $0.94(0.84,1.04)$ & 0.239 & 1.000 \\
rs9468877 & G/A & $1141 / 193 / 7$ & $1718 / 256 / 8$ & 100.00 & $0.08 / 0.07$ & 0.86 & $1.12(0.92,1.35)$ & 0.275 & 1.000 \\
rs1108746 & C/A & $1097 / 233 / 11$ & $1607 / 356 / 19$ & 100.00 & $0.10 / 0.10$ & 1.00 & $0.95(0.80,1.12)$ & 0.541 & 1.000 \\
rs1265156 & C/A & $589 / 579 / 173$ & $835 / 900 / 247$ & 100.00 & $0.34 / 0.35$ & 0.84 & $0.97(0.88,1.08)$ & 0.597 & 1.000 \\
rs887464 & G/A & $487 / 628 / 226$ & $684 / 963 / 335$ & 100.00 & $0.40 / 0.41$ & 0.93 & $0.97(0.88,1.08)$ & 0.625 & 1.000 \\
rs3130503 & G/A & $791 / 485 / 65$ & $1154 / 726 / 102$ & 100.00 & $0.23 / 0.23$ & 0.42 & $0.98(0.87,1.11)$ & 0.773 & 1.000 \\
rs3094188 & A/C & $834 / 452 / 55$ & $1250 / 650 / 82$ & 100.00 & $0.21 / 0.21$ & 0.89 & $1.02(0.90,1.15)$ & 0.775 & 1.000 \\
rs887466 & G/A & $403 / 656 / 281$ & $587 / 981 / 413$ & 99.94 & $0.45 / 0.46$ & 0.93 & $1.00(0.90,1.10)$ & 0.955 & 1.000 \\
\hline
\end{tabular}

${ }^{\mathrm{a}}$ Major/minor allele. ${ }^{\mathrm{b}}$ Major homozygote/heterozygote/rare homozygote between cases and controls. ${ }^{\mathrm{c}}$ Minor allele frequency. ${ }^{\mathrm{d}}$ Hardy-Weinberg equilibrium test among controls.

${ }^{\mathrm{e}}$ Logistic regression with adjustment for age, sex, and pack-year of smoking in additive model.

${ }^{\mathrm{f}} \mathrm{P}$ values of false discovery rate (FDR).

TABLE 3: Associations between 2 functional SNPs in POU5F1 gene with lung cancer risk.

\begin{tabular}{lccc}
\hline Genotype & Case $(N=1,341)$ & Control $(N=1,982)$ & OR $(95 \% \text { CI })^{\mathrm{b}}$ \\
\hline rs887468 $(\mathrm{G}>\mathrm{A})^{\mathrm{a}}$ & & & $P^{\mathrm{b}}$ \\
GG & 997 & 1588 & 1.00 \\
GA & 322 & 368 & $1.33(1.12-1.59)$ \\
AA & 22 & 26 & $1.39(0.78-2.49)$ \\
Additive model & 1341 & 1982 & $1.29(1.11-1.51)$ \\
rs3130457 (A>G) & & & 0.001 \\
AA & 1030 & 1626 & 1.00 \\
AG & 293 & 335 & $1.31(1.10-1.57)$ \\
GG & 18 & 21 & $1.44(0.76-2.76)$ \\
Additive model & 1341 & 1982 & $1.29(1.10-1.51)$ \\
\hline
\end{tabular}

${ }^{\mathrm{a}}$ Major allele > Minor allele. ${ }^{\mathrm{b}}$ Logistic regression with adjustment for age, gender, and pack-years of smoking.

where $Y$ is the logit of case-control status, $G$ and $S$ are factors (SNP or smoking status), $\beta_{0}$ is constant, $\beta_{G}$ and $\beta_{S}$ are the main effects of factors $G$ and $S$, respectively. And $\beta_{G S}$ is the interaction term. Covar ${ }_{i}$ denote covariates for adjustment, including age and sex.

All analyses were performed using $\mathrm{R}$ software (version 3.1.1, The R Foundation for Statistical Computing, http:// www.cran.r-project.org/).

\section{Results}

The geographic characteristics of participants were summarized in Table 1 . The distributions of age $(P=0.980)$ and sex $(P=0.179)$ were comparable between cases and controls. However, cases have larger proportions of smokers and heavy smokers than controls $(P<0.001) .481$ (35.87\%) cases had squamous cell carcinoma and 860 (64.13\%) cases had adenocarcinoma.

The call rates of genotyping for 17 SNPs were all above 98\% (Table 2). The observed genotypes for these SNPs were in agreement with Hardy-Weinberg equilibrium among controls. The variant alleles of 2 SNPs (rs887468 and rs3130457) were significantly associated with increased risk of lung cancer after multiple comparison $(\mathrm{OR}=1.29,95 \% \mathrm{CI}$ : $1.11-$ $1.51, P_{\mathrm{fdr}}=0.017$ for rs887468; OR $=1.29,95 \% \mathrm{CI}: 1.10-1.51$, $P_{\text {fdr }}=0.034$ for rs3130457, resp.) (Tables 2 and 3). However, no significant associations were detected from other SNPs.

To further examine the associations of these 2 SNPs with lung cancer risk, we conducted stratified analyses within subgroups according to selected variables (Table 4). As a whole, the devastating effect of variant alleles was more pronounced in elder age group (>60), males, never smokers, 


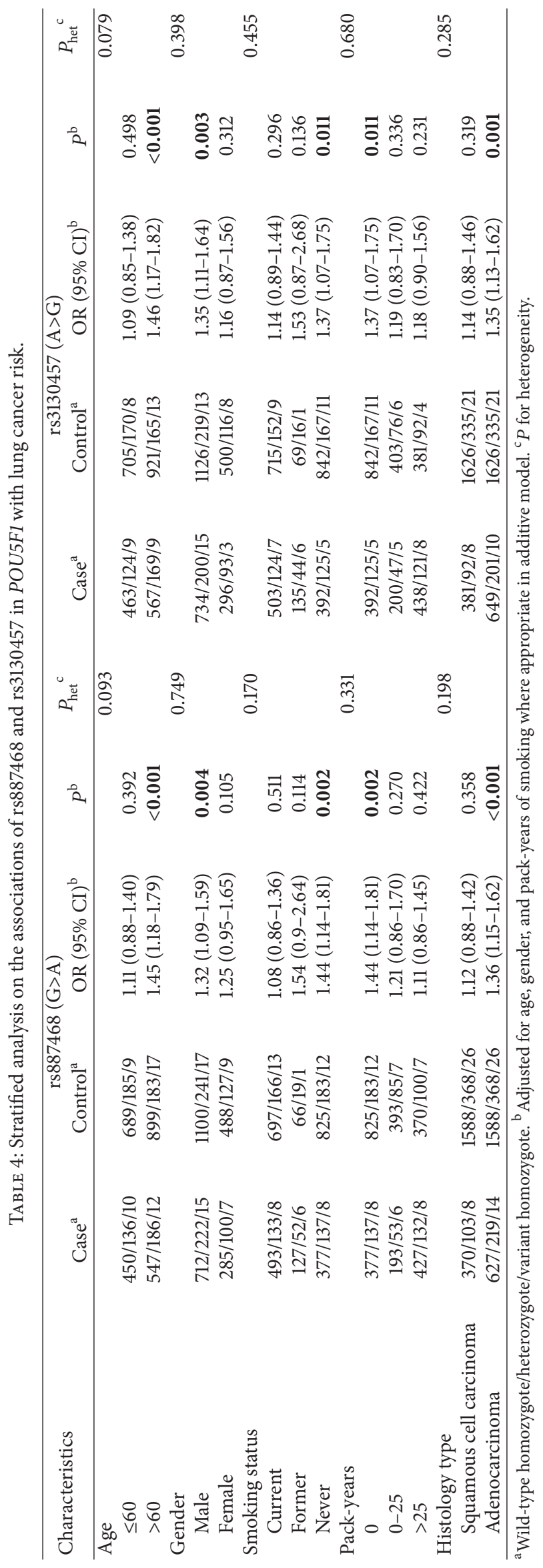


TABLE 5: The interaction between rs 887468 and smoking status on lung cancer risk.

\begin{tabular}{|c|c|c|c|c|c|}
\hline \multirow{2}{*}{ Smoking status } & \multicolumn{3}{|c|}{ rs887468 } & \multirow{2}{*}{ OR $(95 \% \mathrm{CI})^{\mathrm{a}}$} & \multirow{2}{*}{$P^{\mathrm{a}}$} \\
\hline & Genotype & Case & Control & & \\
\hline Nonsmokers & GG & 504 & 891 & 1.00 & \\
\hline Nonsmokers & GA/AA & 203 & 215 & $1.67(1.34-2.08)$ & $<0.001$ \\
\hline Smokers & GG & 493 & 697 & $1.21(1.01-1.46)$ & 0.039 \\
\hline \multirow[t]{2}{*}{ Smokers } & GA/AA & 141 & 179 & $1.35(1.04-1.76)$ & 0.024 \\
\hline & \multicolumn{3}{|c|}{$P$ for multiplicative interaction } & & 0.017 \\
\hline
\end{tabular}

${ }^{a} P$ value of interaction analysis between rs887468 and pack-years of smoking on lung cancer risk with adjustment for age and gender.

and adenocarcinoma patients. No significant heterogeneity was found for ORs and their 95\% CIs between different subgroups. We also explored interaction between these SNPs and smoking status. As shown in Table 5, an interaction existed between the genotype of rs887468 and smoking status ( $P$ for multiplicative interaction $=0.017)$. However, no significant multiplicative interaction was observed for rs3130457 (data not shown). Furthermore, we investigated cumulative effect of these 2 risk alleles on lung cancer and observed significant allele-dosage association between number of risk alleles and lung cancer risk $\left(P_{\text {trend }}<0.001\right)$. People who carried one or two risk alleles were 1.33 times more likely to develop lung cancer than those who carried no risk allele, and those with more than two risk alleles were 1.46 times more likely to develop lung cancer. These data indicated that those who carried more risk alleles had a higher risk of lung cancer.

\section{Discussion}

In the current study, we explored the association of 17 potentially functional SNPs in POU5F1 gene with the development of lung cancer in 1,341 cases and 1,982 healthy controls. We found that the variant allele of rs887468 and rs1310457 were associated with increased lung cancer risk. To the best of our knowledge, it is the first association study of polymorphisms in the POU5F1 gene and lung cancer, which provides more evidence for understanding the role of $P O U 5 F 1$ in lung cancer risk.

POU5F1, a transcription factor, is involved in regulation of pluripotent state of stem cells, and its level decreases with the onset of differentiation in these cells [15-18]. Amini et al. have found that cancer cell lines and cancer tissues had significantly higher expression levels of early embryonic stem cell genes, including POU5F1, SOX2, and CD133 [21]. In the hypoxia conditions, POU5F1 can promote CD133 expression in the lung cancer cells, which is a specific cell surface marker for cancer stem cells [27]. In addition, parallel elevated expression of POU5F1 and Nanog in lung adenocarcinoma (LAC) increases the percentage of CD133-expressing subpopulation, enhances drug resistance, and promotes epithelialmesenchymal transition (EMT); coexpression also activates Slug and enhances the tumor-initiating capability of LAC [28, 29]. Furthermore, knockdown of POU5F1 impeded tumorigenic and metastatic ability and reversed the EMT process of lung adenocarcinoma [28, 29]. For squamous cell carcinoma, Chen et al. measured POU5F1 expression in nonsmall cell lung cancer tissues and found that although the proportion of squamous cell carcinoma tissues which have elevated expression was smaller than that of adenocarcinoma, the increased expression of POU5F1 was associated with poor differentiation of cancer cells and shorter overall survival in both histologic subtype of lung cancer [30]. Taken together, POU5F1 expression is crucial for the selfrenewal and oncogenic potentials of lung cancer stem cells. Our findings further demonstrated that POU5F1 might be a susceptibility gene of lung cancer, which was consistent with the observations mentioned above.

The two SNPs, rs887468 and rs3130457, are located in the upstream region from the transcription starting site of POU5F1 gene. According to a web-based SNP analysis tool, SNPinfo (http://snpinfo.niehs.nih.gov/), the two SNPs are all potential transcriptor binding sites and the SNP rs887468 may influence an exonic splicing enhancer [31] or exonic splicing silencer (ESS) (Supplemental Table 1) (see Supplementary Material available online at http://dx.doi.org/10.1155/ 2015/851320). Variations in ESE or ESS can be involved in disruptions of balanced interplay of ESE- and ESS-binding proteins, which thereby results in missplicing and causes deficiency in expression products of nearby genes [31-34]. We speculate that variant genotype of rs887468 might lead to alternative splicing events and disequilibrium for different isoforms of POU5F1, suggesting a biological plausible mechanism for lung cancer risk. Variation in rs887468 may influence its interactions with transcription factors such as Myc-associated zinc-finger protein (MAZ) (as predicted by RegulomeDB (http://www.regulomedb.org/)). MAZ gene normally expresses in the lungs [35]. It is responsible for regulation of oncogene transcription from promoter [36]. One possible explanation for the association between rs887468 and lung cancer risk might be that the variant genotype alters interactions of the loci with transcription factors and results in aberration in function of $P O U 5 F 1$ gene and elicits procedure of carcinogenesis.

Based on an online tool, DNase-seq and RegulomeDB (http://www.regulomedb.org/), rs887468 and rs3130457 fall into DNase I peaks. DNase I hypersensitivity regions are potential genetic regulatory loci and correlate with binding sites of sequence-specific DNA-binding proteins [37]. This suggests a possible mechanism for the effect of these variants on lung cancer risk. Additionally, a multiplicative interaction was observed between rs887468 in POU5F1 gene and smoking status. The interaction result indicated that the harmful effect of variant allele of rs887468 was weaker among current smokers. However, our results are very preliminary; further 
experimental studies are warranted to uncover the underling mechanism of these observations.

In summary, our case-control study reported 2 potential functional SNPs in POU5F1 gene that may affect the risk for lung cancer in a Chinese population. Given that our results are very preliminary, more large scale studies are required to validate our findings in diverse ethnic populations and clarify the molecular basis behind these observations.

\section{Conflict of Interests}

The authors declare that there is no conflict of interests regarding the publication of this paper.

\section{Authors' Contribution}

Rui Niu, Yuzhuo Wang, and Meng Zhu contributed equally to this work.

\section{Acknowledgments}

This work was funded by the State Key Program of National Natural Science of China (81230067), the National Key Basic Research Program Grant (2011CB503805), the National Science and Technology Support Program (2011BAI09B02), National Outstanding Youth Science Foundation of China (81225020), National Natural Science of China (81573238, 81521004, 81270044, and 81302488), National Program for Support of Top-Notch Young Professionals from the Organization Department of the CPC Central Committee, Science Foundation for Distinguished Young Scholars of Jiangsu (BK2012042), Jiangsu Province Clinical Science and Technology Projects (BL2012008), and the Priority Academic Program for the Development of Jiangsu Higher Education Institutions [Public Health and Preventive Medicine]. The authors wish to thank all the study participants, research staff, and students who participated in this work.

\section{References}

[1] D. M. Parkin, P. Pisani, A. D. Lopez, and E. Masuyer, "At least one in seven cases of cancer is caused by smoking. Global estimates for 1985," International Journal of Cancer, vol. 59, no. 4, pp. 494-504, 1994.

[2] C. D. Berg, D. R. Aberle, and D. E. Wood, "Lung cancer screening: promise and pitfalls," American Society of Clinical Oncology Educational Book, pp. 450-457, 2012.

[3] C. I. Amos, X. Wu, P. Broderick et al., "Genome-wide association scan of tag SNPs identifies a susceptibility locus for lung cancer at 15q25.1," Nature Genetics, vol. 40, no. 5, pp. 616-622, 2008.

[4] P. Broderick, Y. Wang, J. Vijayakrishnan et al., "Deciphering the impact of common genetic variation on lung cancer risk: a genome-wide association study," Cancer Research, vol. 69, no. 16, pp. 6633-6641, 2009.

[5] J. Dong, Z. Hu, C. Wu et al., "Association analyses identify multiple new lung cancer susceptibility loci and their interactions with smoking in the Chinese population," Nature Genetics, vol. 44, no. 8, pp. 895-899, 2012.
[6] Z. Hu, C. Wu, Y. Shi et al., "A genome-wide association study identifies two new lung cancer susceptibility loci at 13q12.12 and 22q12.2 in Han Chinese," Nature Genetics, vol. 43, no. 8, pp. 792796, 2011.

[7] R. J. Hung, J. D. McKay, V. Gaborieau et al., "A susceptibility locus for lung cancer maps to nicotinic acetylcholine receptor subunit genes on 15q25," Nature, vol. 452, no. 7187, pp. 633-637, 2008.

[8] Q. Lan, C. A. Hsiung, K. Matsuo et al., "Genome-wide association analysis identifies new lung cancer susceptibility loci in never-smoking women in Asia," Nature Genetics, vol. 44, pp. 1330-1335, 2012.

[9] J. D. McKay, R. J. Hung, V. Gaborieau et al., "Lung cancer susceptibility locus at 5p15.33," Nature Genetics, vol. 40, no. 12, pp. 1404-1406, 2008.

[10] K. Shiraishi, H. Kunitoh, Y. Daigo et al., "A genome-wide association study identifies two new susceptibility loci for lung adenocarcinoma in the Japanese population," Nature Genetics, vol. 44, no. 8, pp. 900-903, 2012.

[11] Y. Wang, P. Broderick, E. Webb et al., "Common 5p15.33 and 6p21.33 variants influence lung cancer risk," Nature Genetics, vol. 40, no. 12, pp. 1407-1409, 2008.

[12] Y. Babaie, R. Herwig, B. Greber et al., "Analysis of Oct4dependent transcriptional networks regulating self-renewal and pluripotency in human embryonic stem cells," STEM CELLS, vol. 25, no. 2, pp. 500-510, 2007.

[13] H. R. Schöler, S. Ruppert, N. Suzuki, K. Chowdhury, and P. Gruss, "New type of POU domain in germ line-specific protein Oct-4," Nature, vol. 344, no. 6265, pp. 435-439, 1990.

[14] K. Okamoto, H. Okazawa, A. Okuda, M. Sakai, M. Muramatsu, and H. Hamada, "A novel octamer binding transcription factor is differentially expressed in mouse embryonic cells," Cell, vol. 60, no. 3, pp. 461-472, 1990.

[15] M. Pesce and H. R. Schöler, "Oct-4: gatekeeper in the beginnings of mammalian development," Stem Cells, vol. 19, no. 4, pp. 271-278, 2001.

[16] M. H. Rosner, M. A. Vigano, K. Ozato et al., "A POU-domain transcription factor in early stem cells and germ cells of the mammalian embryo," Nature, vol. 345 , no. 6277, pp. 686-692, 1990.

[17] M. Monk and C. Holding, "Human embryonic genes reexpressed in cancer cells," Oncogene, vol. 20, no. 56, pp. 80858091, 2001.

[18] M.-H. Tai, C.-C. Chang, L. K. Olson, and J. E. Trosko, "Oct4 expression in adult human stem cells: evidence in support of the stem cell theory of carcinogenesis," Carcinogenesis, vol. 26, no. 2, pp. 495-502, 2005.

[19] J. E. Visvader and G. J. Lindeman, "Cancer stem cells in solid tumours: accumulating evidence and unresolved questions," Nature Reviews Cancer, vol. 8, no. 10, pp. 755-768, 2008.

[20] U. R. Rapp, F. Ceteci, and R. Schreck, "Oncogene-induced plasticity and cancer stem cells," Cell Cycle, vol. 7, no. 1, pp. 4551, 2008.

[21] S. Amini, F. Fathi, J. Mobalegi, H. Sofimajidpour, and T. Ghadimi, "The expressions of stem cell markers: Oct4, Nanog, Sox2, nucleostemin, Bmi, Zfx, Tcl1, Tbx3, Dppa4, and Esrrb in bladder, colon, and prostate cancer, and certain cancer cell lines," Anatomy \& Cell Biology, vol. 47, no. 1, pp. 1-11, 2014.

[22] S. Gidekel, G. Pizov, Y. Bergman, and E. Pikarsky, "Oct-3/4 is a dose-dependent oncogenic fate determinant," Cancer Cell, vol. 4, no. 5, pp. 361-370, 2003. 
[23] C.-C. Chang, G.-S. Shieh, P. Wu, C.-C. Lin, A.-L. Shiau, and C.-L. Wu, "Oct-3/4 expression reflects tumor progression and regulates motility of bladder cancer cells," Cancer Research, vol. 68, no. 15, pp. 6281-6291, 2008.

[24] G. Karoubi, L. Cortes-Dericks, M. Gugger, D. Galetta, L. Spaggiari, and R. A. Schmid, "Atypical expression and distribution of embryonic stem cell marker, OCT4, in human lung adenocarcinoma," Journal of Surgical Oncology, vol. 102, no. 6, pp. 689-698, 2010.

[25] L. Shen, M. Du, C. Wang et al., "Clinical Significance of POU5F1P1 rs10505477 polymorphism in Chinese gastric cancer patients receving cisplatin-based chemotherapy after surgical resection," International Journal of Molecular Sciences, vol. 15, no. 7, pp. 12764-12777, 2014.

[26] M. M. Finucane, G. A. Stevens, M. J. Cowan et al., "National, regional, and global trends in body-mass index since 1980: systematic analysis of health examination surveys and epidemiological studies with 960 country-years and 9.1 million participants," The Lancet, vol. 377, no. 9765, pp. 557-567, 2011.

[27] H. Iida, M. Suzuki, R. Goitsuka, and H. Ueno, "Hypoxia induces CD133 expression in human lung cancer cells by up-regulation of OCT3/4 and SOX2," International Journal of Oncology, vol. 40, no. 1, pp. 71-79, 2012.

[28] S.-H. Chiou, M.-L. Wang, Y.-T. Chou et al., "Coexpression of Oct4 and Nanog enhances malignancy in lung adenocarcinoma by inducing cancer stem cell-like properties and epithelialmesenchymal transdifferentiation," Cancer Research, vol. 70, no. 24, pp. 10433-10444, 2010.

[29] C. Xu, D. Xie, S.-C. Yu et al., “ $\beta$-catenin/POU5F1/SOX2 transcription factor complex mediates IGF-I receptor signaling and predicts poor prognosis in lung adenocarcinoma," Cancer Research, vol. 73, no. 10, pp. 3181-3189, 2013.

[30] Z. Chen, T. Wang, L. Cai et al., "Clinicopathological significance of non-small cell lung cancer with high prevalence of Oct4 tumor cells," Journal of Experimental and Clinical Cancer Research, vol. 31, article 10, 2012.

[31] R. K. Olsen, S. Broner, R. Sabaratnam et al., "The ETFDH c. $158 \mathrm{~A}>\mathrm{G}$ variation disrupts the balanced interplay of ESE- and ESS-binding proteins thereby causing missplicing and multiple Acyl-CoA dehydrogenation deficiency," Human Mutation, vol. 35, pp. 86-95, 2014.

[32] A. Nishida, N. Kataoka, Y. Takeshima et al., "Chemical treatment enhances skipping of a mutated exon in the dystrophin gene," Nature Communications, vol. 2, no. 1, article 308, 2011.

[33] S. Sen, I. Talukdar, and N. J. G. Webster, "SRp20 and CUGBP1 modulate insulin receptor exon 11 alternative splicing," Molecular and Cellular Biology, vol. 29, no. 3, pp. 871-880, 2009.

[34] B. J. Blencowe, "Exonic splicing enhancers: mechanism of action, diversity and role in human genetic diseases," Trends in Biochemical Sciences, vol. 25, no. 3, pp. 106-110, 2000.

[35] J. Song, H. Murakami, H. Tsutsui et al., "Genomic organization and expression of a human gene for Myc-associated zinc finger protein (MAZ)," The Journal of Biological Chemistry, vol. 273, no. 32, pp. 20603-20614, 1998.

[36] L. Jiao, Y. Li, D. Shen et al., “The prostate cancer-up-regulated myc-associated zinc-finger protein (MAZ) modulates proliferation and metastasis through reciprocal regulation of androgen receptor," Medical Oncology, vol. 30, no. 2, article 570, 2013.

[37] L. C. Murphy, N. Huzel, and J. R. Davie, "Novel DNase I hypersensitive sites in the $3^{\prime}$-flanking region of the human cmyc gene," DNA and Cell Biology, vol. 15, no. 7, pp. 543-548, 1996. 

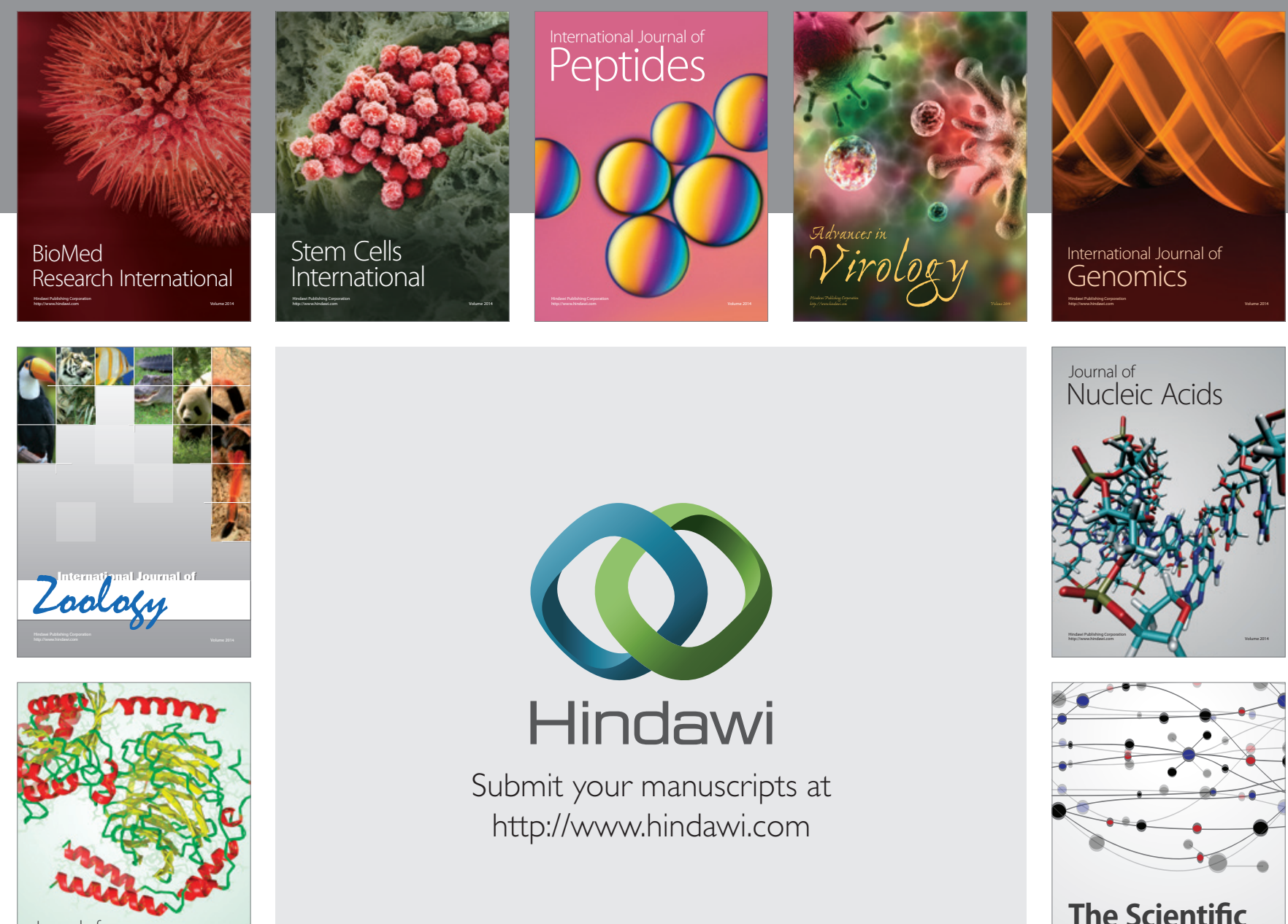

Submit your manuscripts at

http://www.hindawi.com

Journal of
Signal Transduction
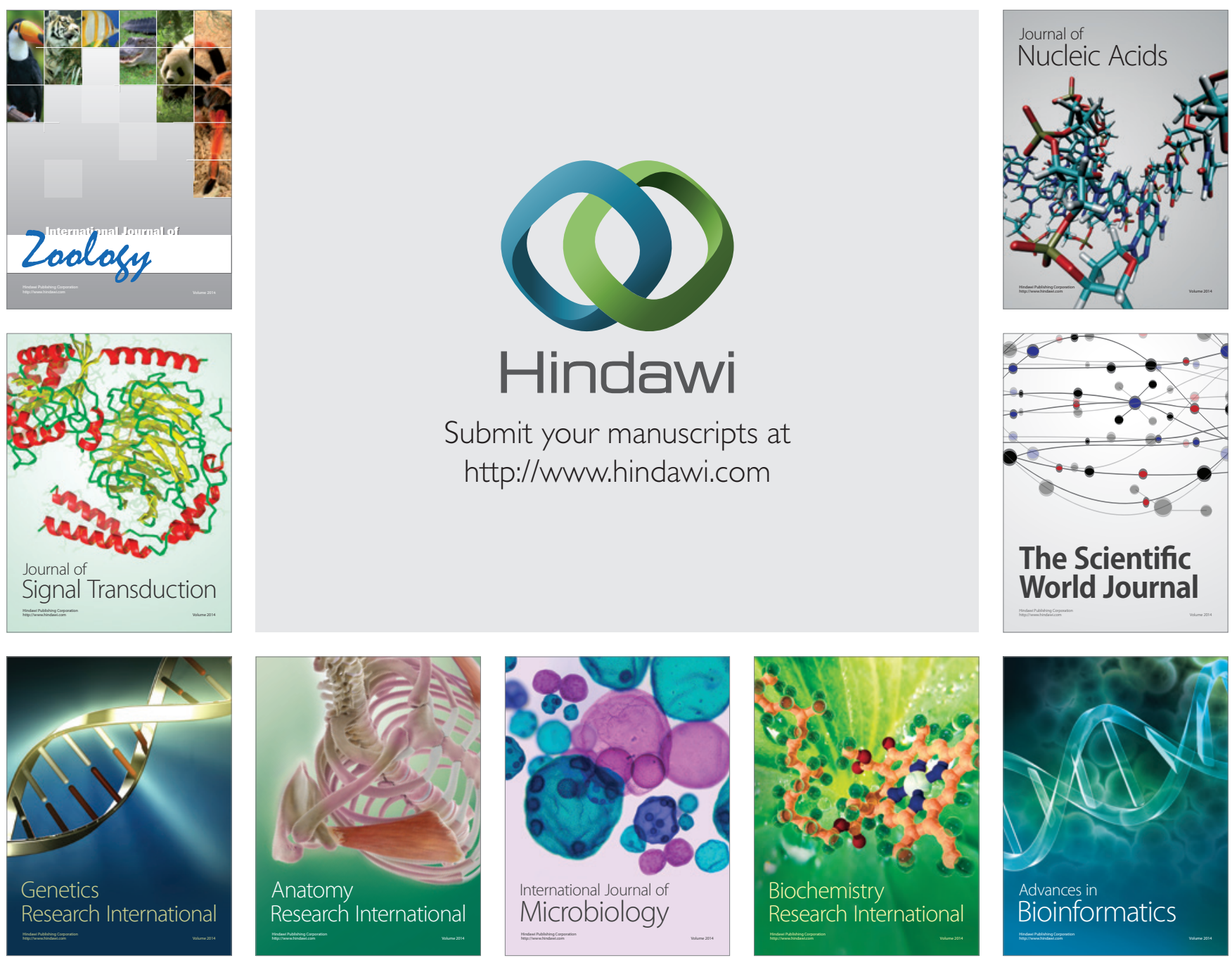

The Scientific World Journal
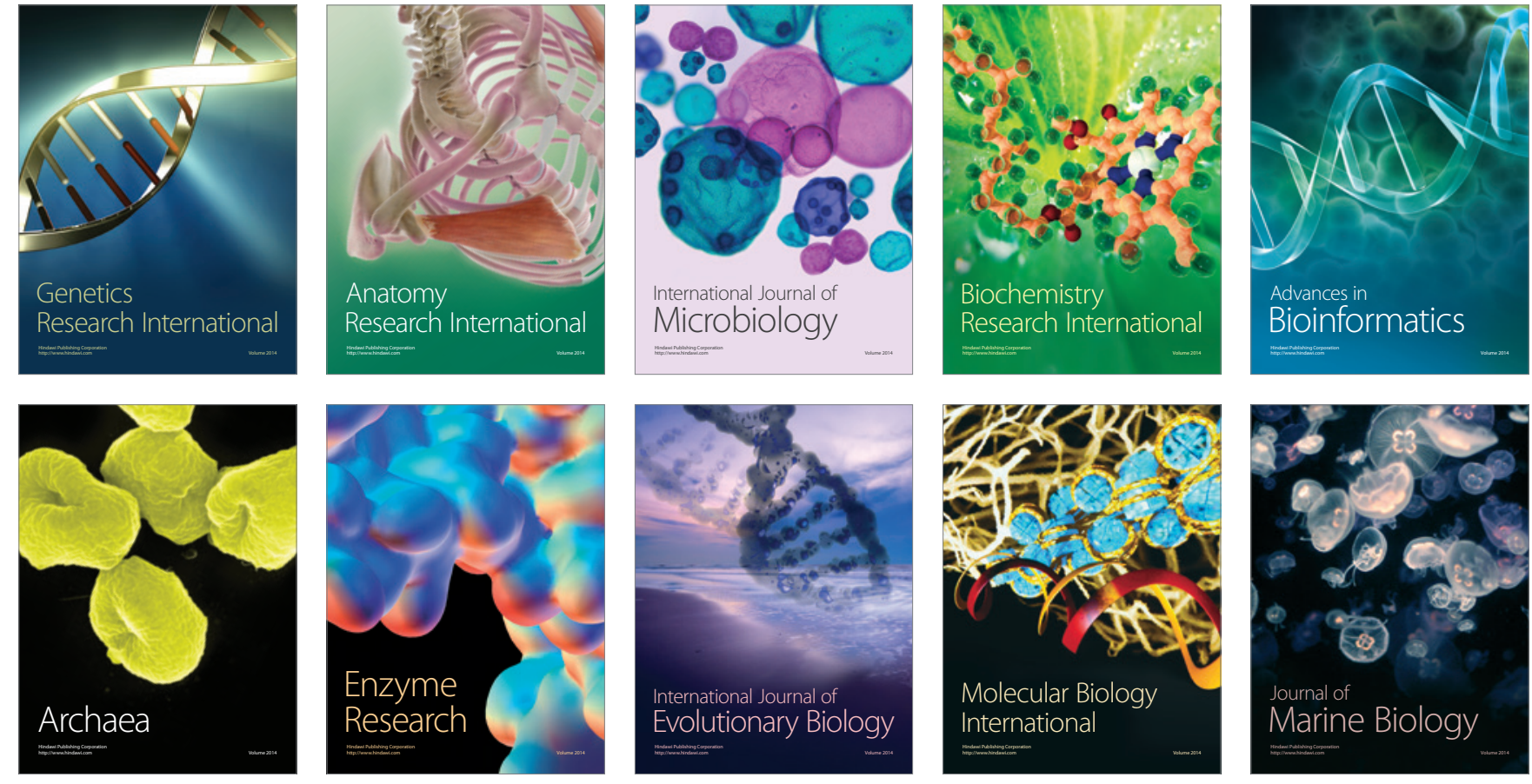